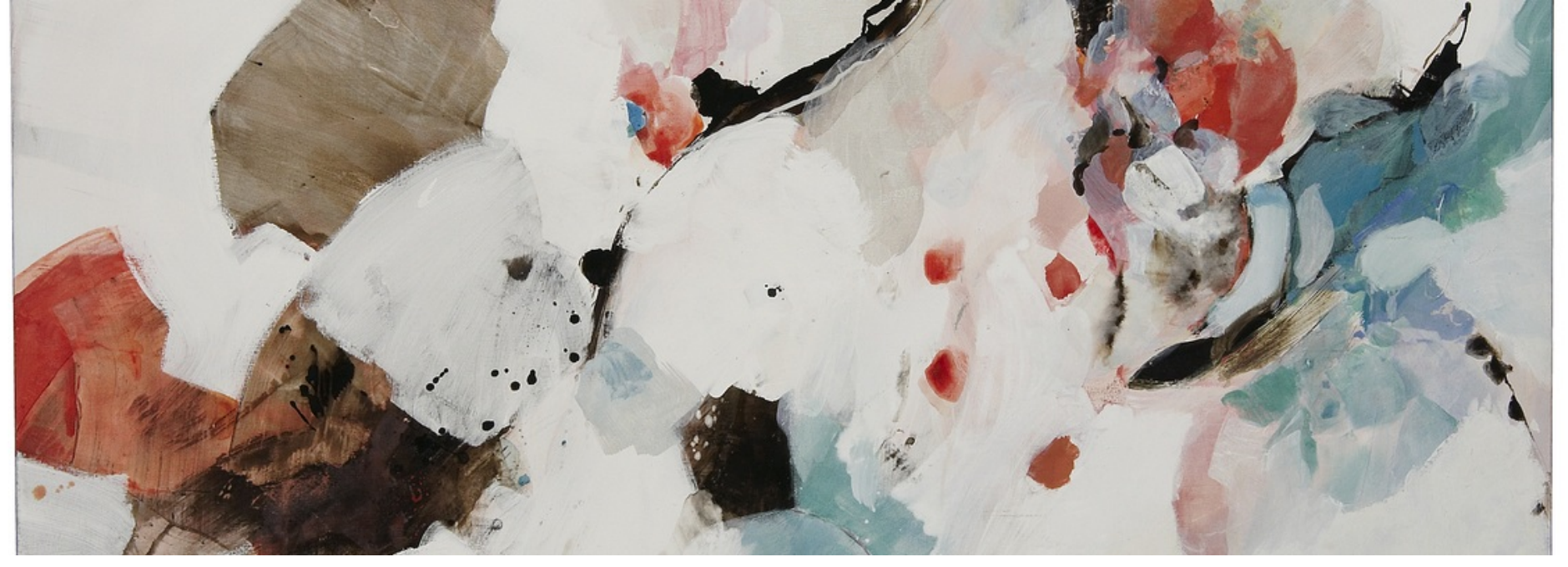

\title{
La peinture nitescente
}

\section{Authors: Albane Roux}

Submitted:

15. November 2015

Published:

Volume:

Issue:

15. November 2015

Keywords:

$$
2
$$

7

DOI: $\quad$ 10.17160/josha.2.7.80 


\section{ALBANE ROUX}

\section{La peinture nitescente}

Le travail d'Albane Roux s'attache à la peinture, mais son geste plastique s'apparente plus à celui du sculpteur qu'à celui du peintre. En effet, chaque pose de matière sur la toile n'est pas pour elle destinée seulement à produire une touche de couleur, à créer une graphie chromatique ayant une valeur tactile, cinétique ou expressive, mais d'abord un geste. Un geste, c'est-à-dire ni une expression ou une action mais un mouvement intérieur qui permet de retrouver " quelque chose " de la constitution d'un sujet, d'une singularité oeuvrante. Le geste porte la chose à l'expérience de la perception, au-delà d'un désir de représentation du réel il fait advenir au monde l'infigurable. En ce sens la peinture d' Albane Roux se tient au-delà du débat un peu stérile entre figuration et non figuration, car l'abstraction pour elle est un espace vivant, un lieu d'expériences ouvert sur le monde.

Olivier Debré a formalisé sous le terme d' " abstraction fervente ", une intention picturale qui, audelà de la question de la représentation, s'attache hors de la réalité visible à " traduire la sensation " éprouvée devant un être ou dans la nature. Cette démarche n'est pas abstraite, les signes qu'elle engendre signifient la réalité, non telle qu'elle se présente à la vue, mais telle que l'artiste l'éprouve dans son for intérieur. II déclare : " Je me défends d'être un paysagiste, je traduis l'émotion qui est en moi devant le paysage, mais pas le paysage. " La peinture d'Albane Roux s'inscrit dans cette esthétique.

La tradition picturale qui anime sa création est celle de la nouvelle École de Paris qui triomphe dans les années 1950, avec, outre Olivier Debré, Hans Hartung, Nicolas de Staël, Zao Wou ki, Pierre Soulages, etc. Le travail pictural de Zao Wou ki; est par bien des manières en filiation directe avec celui d'Albane Roux, sensible aux passages entre orient et occident. En effet, les formes picturales qui animent ses tableaux empruntent implicitement à ces deux cultures.

La trajectoire de création d'Albane Roux, est marquée par trois moments qui correspondent à deux processus de création bien distincts qui peuvent se côtoyer dans sa production. Le premier processus, à partir d'une image de mémoire, par exemple : un « poilu » de la guerre 14-18, des mariés, une communiante, etc., conduit le geste de l'artiste à " voiler par le blanc » à « évider par le blanc » en recouvrant de peinture les figures présentes sur l'image. Seuls, subsistent des détails visibles pour le spectateur, des mains, une oreille, un bout de chaussure verni ... L'image première, figurative, stéréotypée, est devenue invisible, enfouie dans l'image seconde. Peindre constitue un geste rageur, un geste de sculpture. « Je construis parce que je détruis » dit-elle. Le second processus concerne la période des " Paysages ". Albane Roux décrit de la manière suivante sa genèse : "Quand il n'y a plus eu de séparation entre le dehors et le dedans, j'ai pu entrer dans la diagonale et trouver un mouvement unificateur, qui rassemble, qui réunit tous les éléments construisant l'unité de la toile ». Tout d'abord, "Choisir le moment de peinture et à partir du ressenti, capter ce qui se passe », puis « Poser une couleur, la regarder vivre, y en associer une autre».

Le moment « paysages » m'a incité à passer au grand format. « Paysage et intérieur du corps sont décrits de la même façon, dedans dehors deviennent les mêmes ... Je peins toutes les peaux, les enveloppes du corps ... M'enfouir toujours plus loin, laisser des mues sur mon passage ... Les textures de mes peintures ressemblent à des écailles ou à de fines pellicules blanches de tendresse. Le mouvement sur la toile c'est la vie ». Albane Roux aime l'emploi du goudron; le noir ponctue dans ses toiles, les couleurs et le blanc iridescent. Le goudron est le matériau privilégié qui l'a incitée à l'emploi du noir, d'abord opaque avec le goudron à l'eau, puis jouant de la transparence des glacis 
avec le goudron à l'huile. Elle utilise également la cire de carnauba, un matériau écologique issu des feuilles d'un palmier du Brésil. Paradoxe et liberté de l'artiste qui trouve dans le vivant les éléments nécessaires à sa création.

La peinture d'Albane Roux est une respiration, un mouvement qui invite le spectateur à un voyage intérieur ouvert sur le monde.

Texte de Christian Malaurie, poète, essayiste, critique d'art, écrit en Septembre 2013

\begin{abstract}
Albane Roux en été 2013:
"Le résultat, l'objet tableau ne m'intéresse pas "

"C'est toujours le même tableau que je peins et repeins "

"Je déteste amonceler mes toiles. Je repeins par-dessus"

Dans l'histoire de la peinture gestuelle nous sommes passés de la calligraphie chinoise au geste héroïque américain (action painting), de la radicalité japonaise à la valorisation moderne du processus. Un art mettant en avant « le sens de l'ordinaire » à partir de l'existence vécue et de la quotidienneté.
\end{abstract}

C'est précisément de cette logique instrumentale de la communication que le geste cherche à se dégager, étant donné qu'elle réduit l'oeuvre et l'art à un moyen pour dire quelque chose, et l'autre à un sujet-récepteur. Le geste augurait dans l'oeuvre s'inscrirait là où les individus sont en rapport les uns aux autres avant même d'en avoir décidé. Dire alors que l'oeuvre par ce geste parle à partir de cette forme ouverte de communication, $c^{\prime}$ est dire qu'elle ne communique pas du sens mais qu'elle se communique elle-même comme sens.

C'est même lorsque toute intention de communiquer du sens se suspend que la communication devient possible et que le sens se donne, un sens qui résonne ici de l'en commun. Ce qui se donne alors n'est plus du sens mais le partage. L'expansion d'un geste passant de l'ascèse de la calligraphie chinoise au geste héroïque américain, de la radicalité japonaise à la valorisation moderne du processus. Le geste nous revient de cette histoire où la forme de l'oeuvre naissait « d'un ressassement de la surface par le geste. »

Mais ce geste ressassant ne doit pas oublier non plus de se modeler sur l'évolution immatérielle qui a bouleversé l'oeuvre à l'ère contemporaine. Et voir dans quelle mesure ce geste peut, enrichi de sa tradition picturale, s'annoncer comme une action pure. Ainsi donc, nous tenterons d'envisager le geste, maintenu dans une opposition à la performance, non pas comme un acte à accomplir ou à endurer, mais ancré dans le face-à-face avec l'autre. ce geste, que s'il a « son lieu de nomination - au centre du langage - là où s'engendre temporellement le figurable ", se profile derrière toutes opérations verbales, picturales, musicales, chorégraphiques, artistiques, lui qui est " puissance de ce qu'il bouge et mobilise dans le retrait de toute réalisation, de toute expression. »

Les oeuvres d'art traduisent toujours un rapport entre l'observateur et l'observé.

C'est à partir de la virtualité du geste que toute véritable technique est un inédit à réinventer et non un préalable à appliquer, comme on le voit trop souvent dans les observations.

La problématique du geste est condition d'écoute au-delà du descriptif et de l'observable prônés par les objectivations de notre époque qui font usage d'une multiplicité de notions palliatives. 


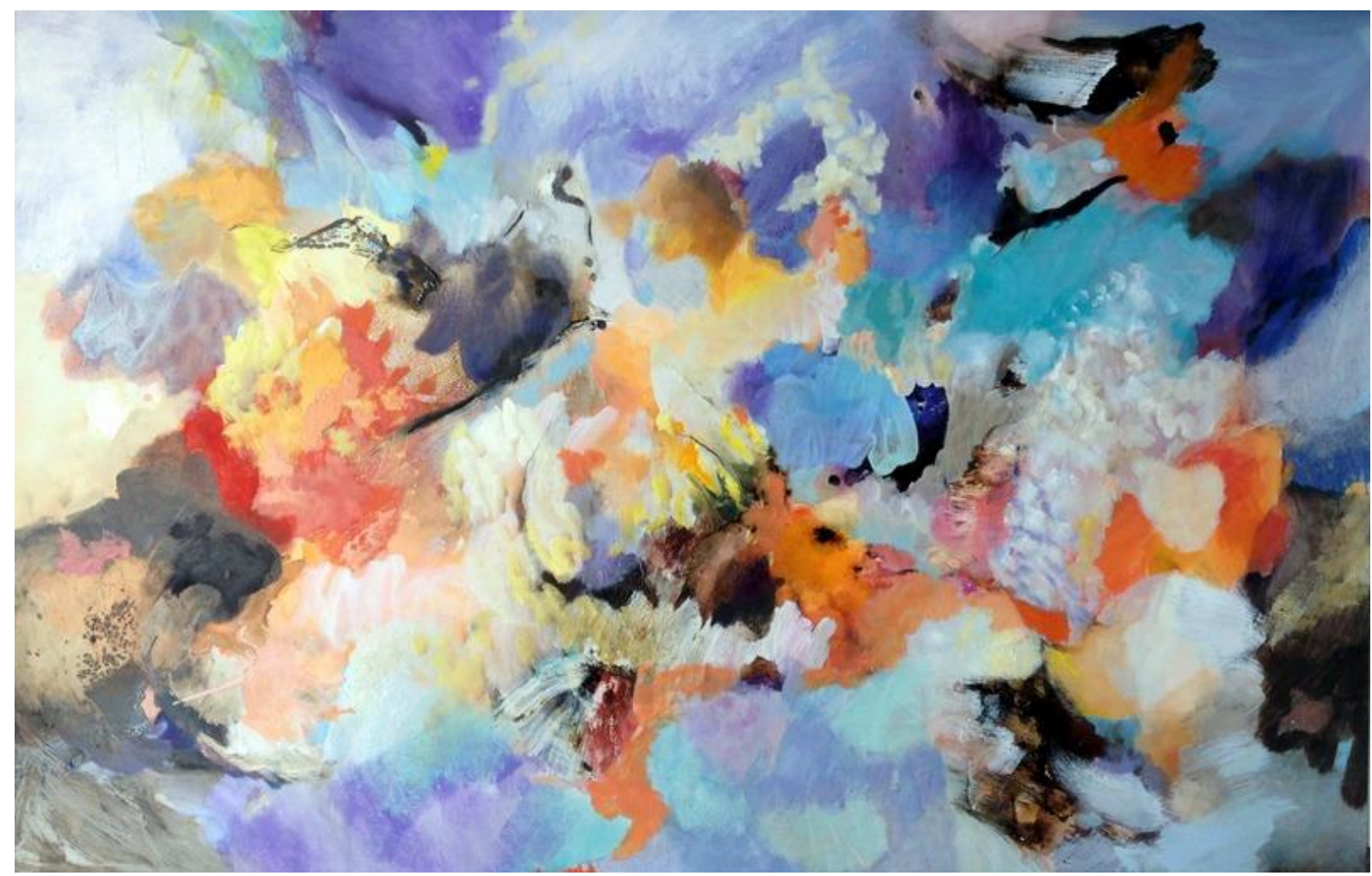

Eau sentimentale 2015

Huile sur toile, $130 \times 195 \mathrm{~cm}$ 


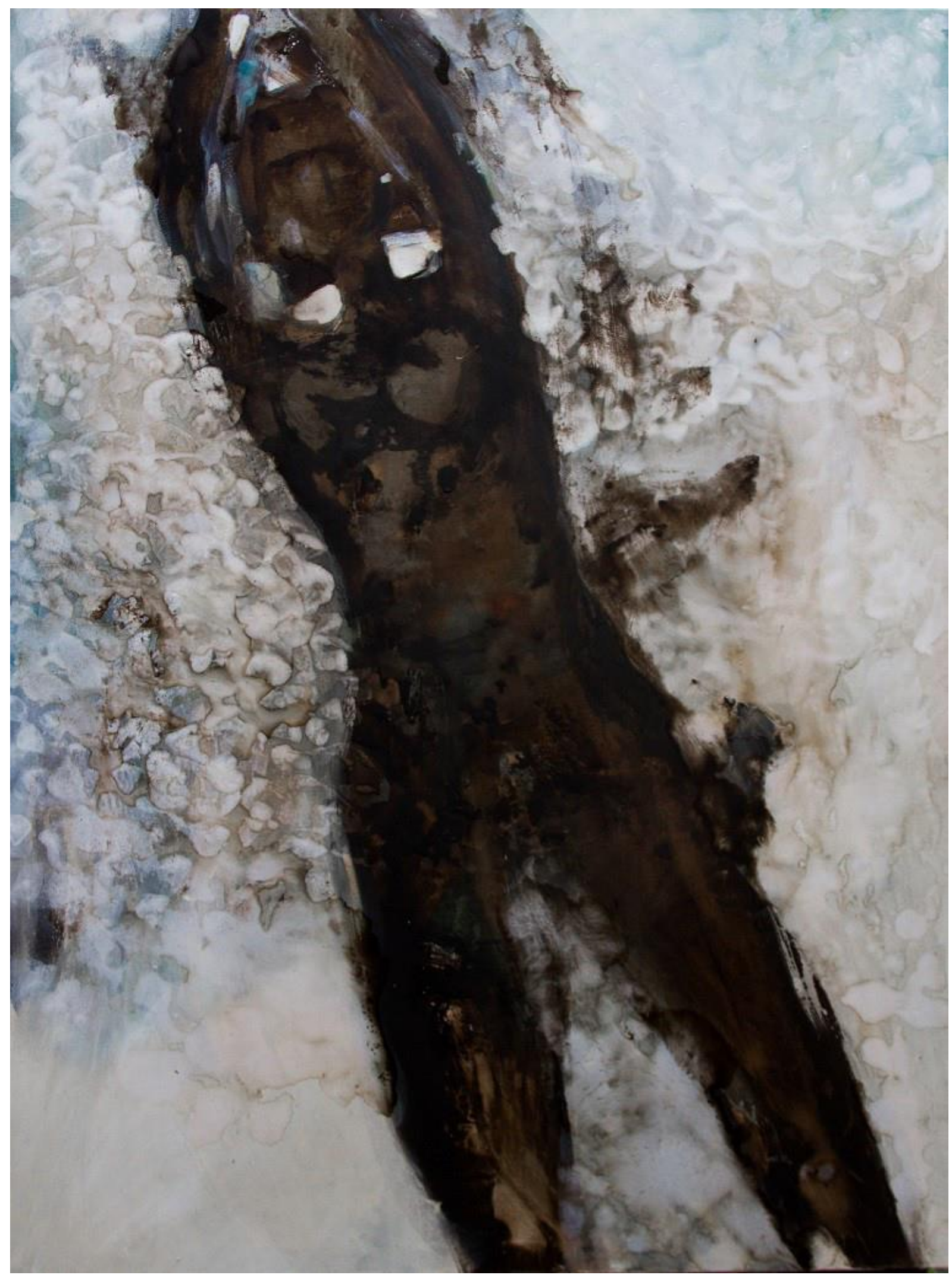

L'être blanc 2013

Technique mixte sur toile $130 \times 195 \mathrm{~cm}$ 


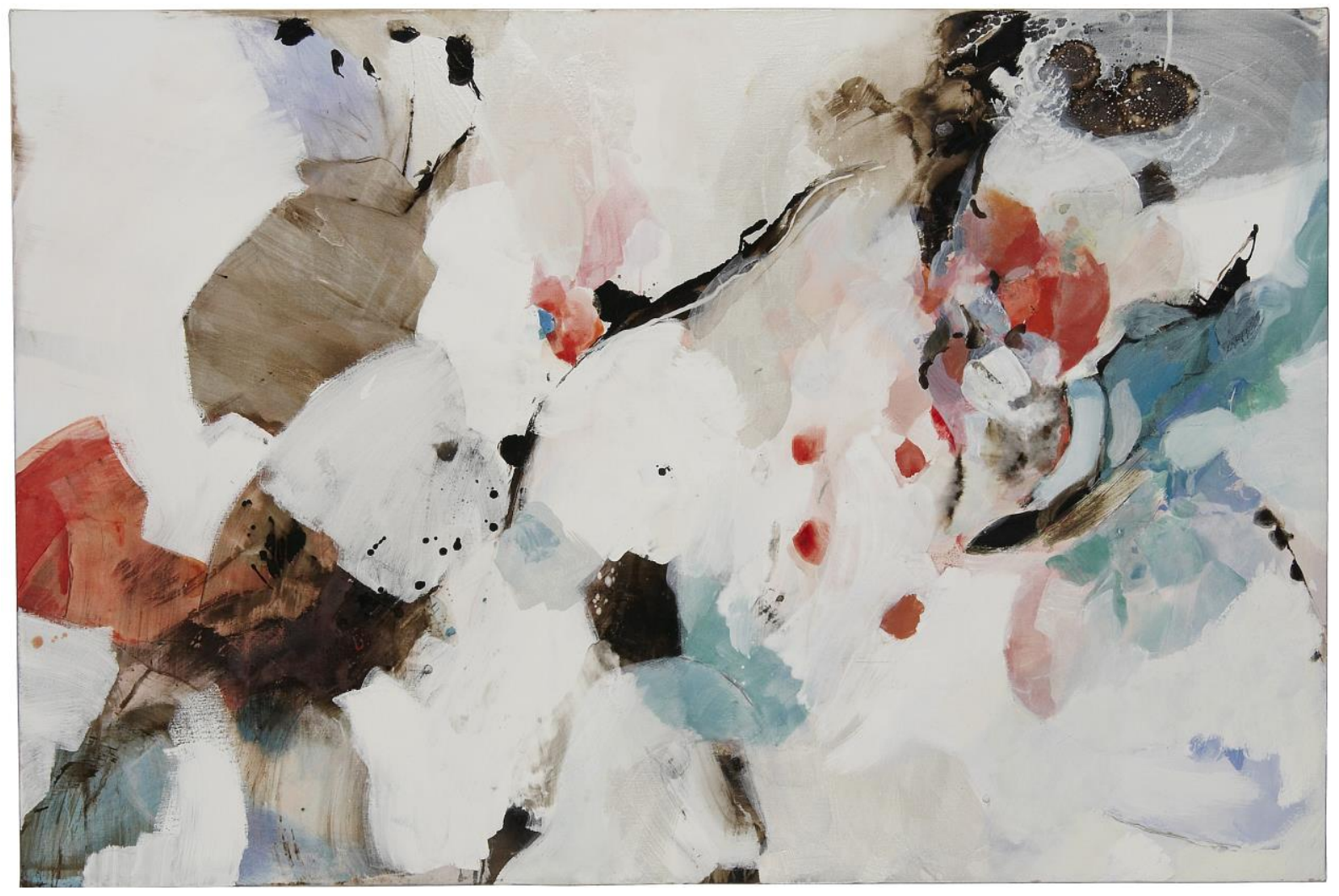

\section{Pans 2013}

Technique mixte sur toile $130 \times 195 \mathrm{~cm}$ 


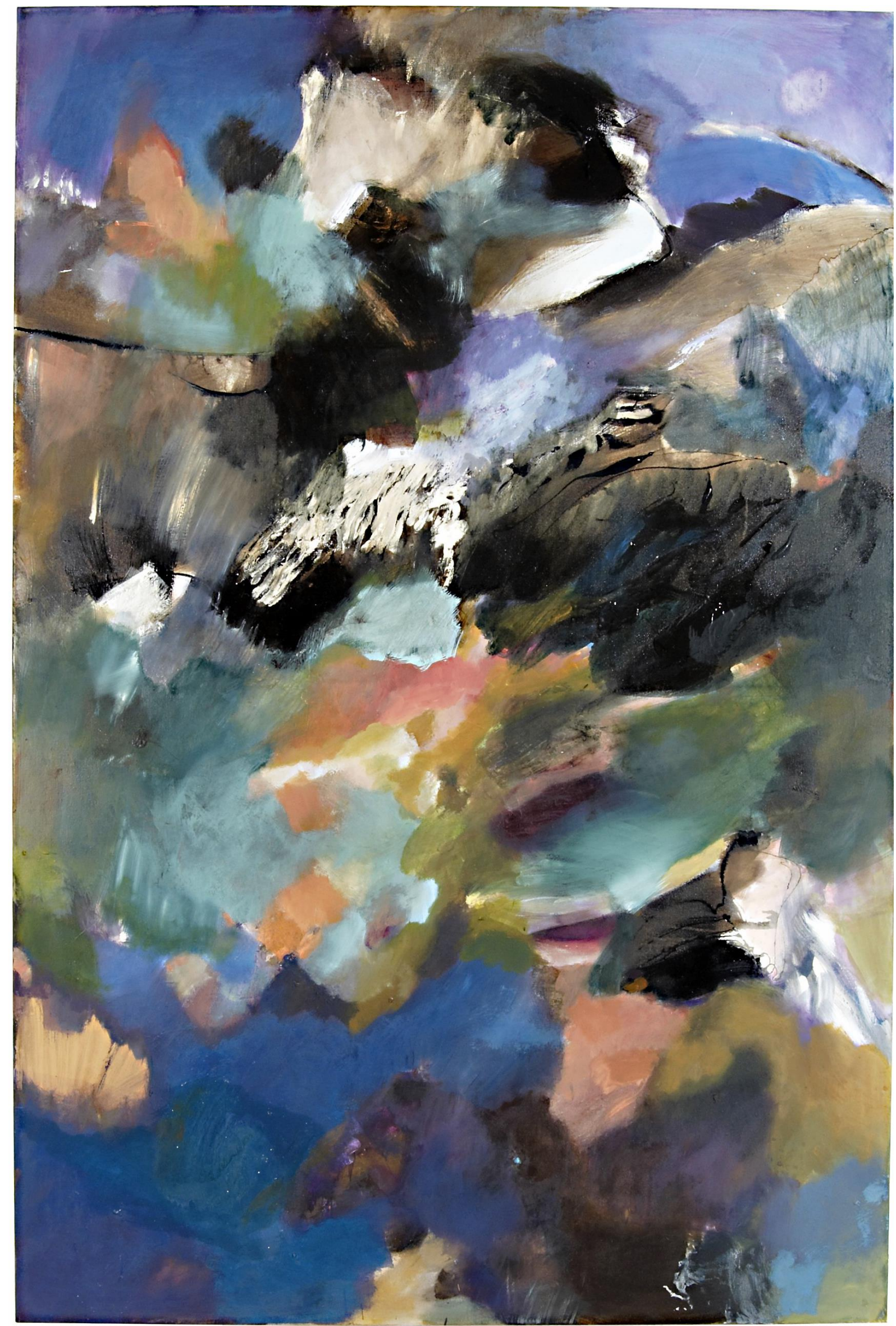

Tamas 2014

Technique mixte sur toile $130 \times 195 \mathrm{~cm}$ 


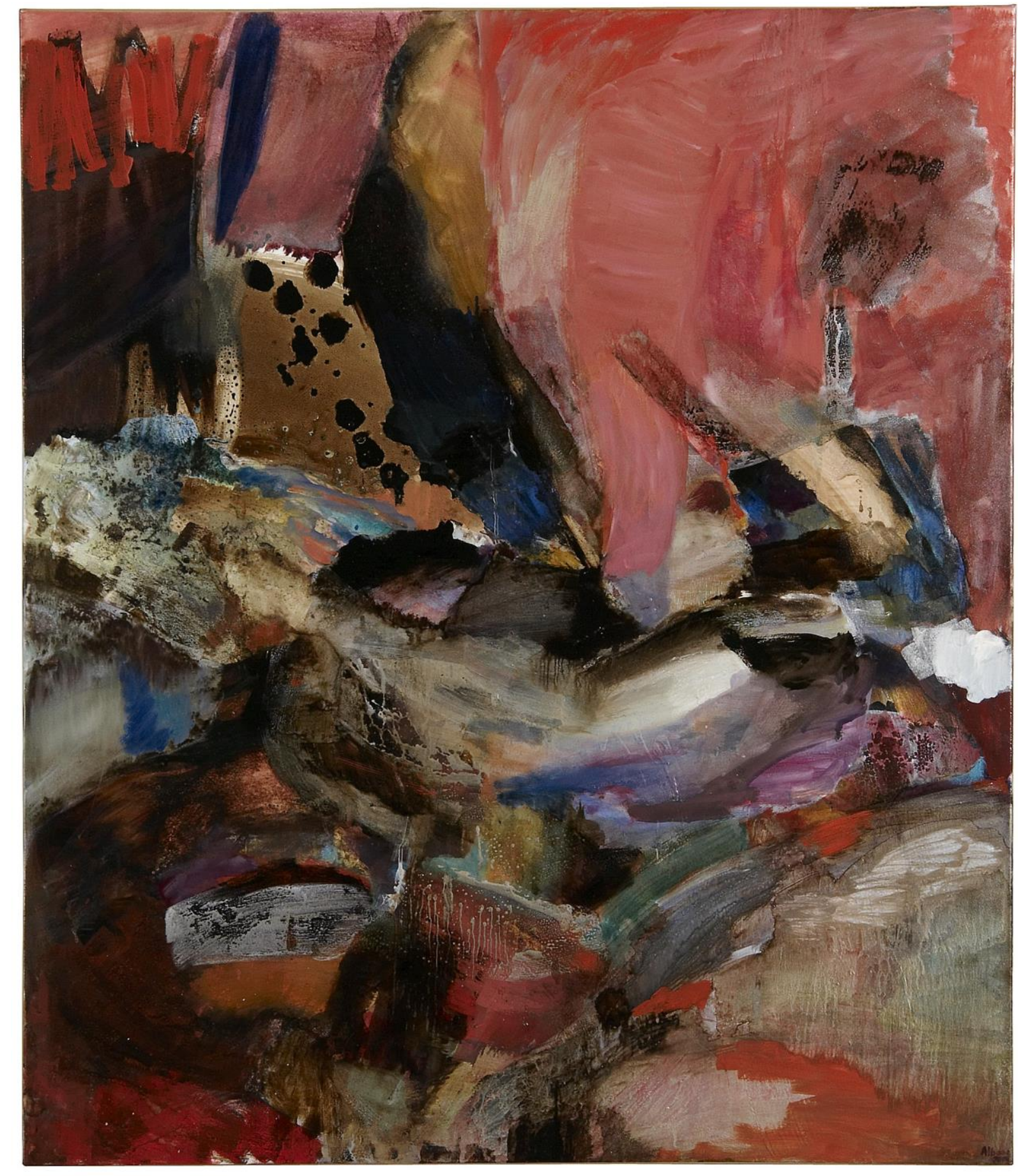

Nuits mauves 2014

Huile sur toile $100 \times 120 \mathrm{~cm}$ 


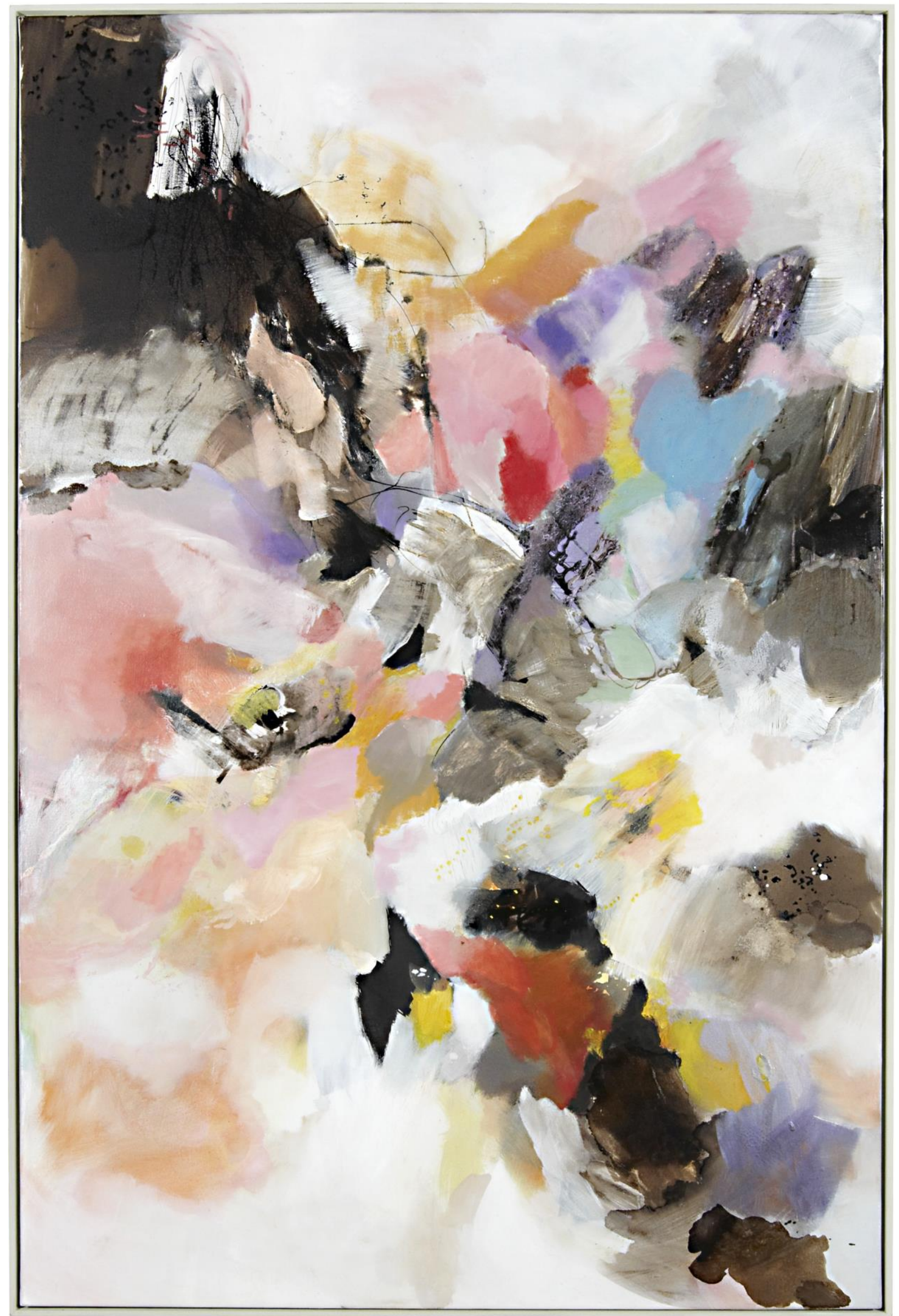

Rajas 2014

Technique mixte sur toile $130 \times 195 \mathrm{~cm}$ 


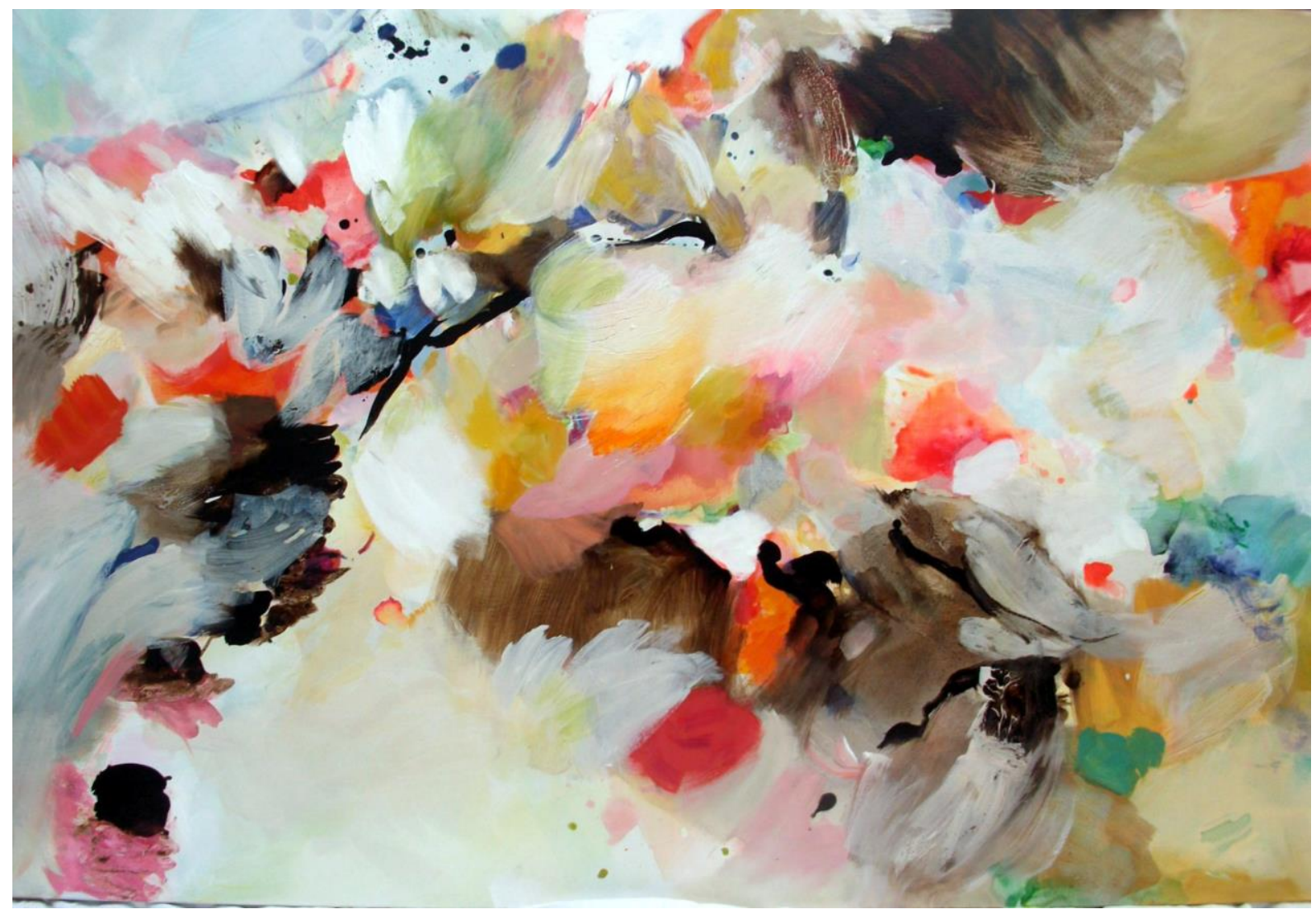

Capture 2014

Technique mixte sur toile $130 \times 195 \mathrm{~cm}$ 